\title{
Rare Case of Rectal Mixed Adeno-Neuroendocrine Carcinoma
}

\author{
Aleksander Khudiakov ${ }^{1}$,Mohammed Al-Sadawi ${ }^{1}$, Jia Qin ${ }^{1}$, Michael Haddadin ${ }^{1}$, \\ Stanley Soroka ${ }^{1}$, Angeleque Hartt ${ }^{1}$, Lina Soni ${ }^{1}$, Shalini Arora ${ }^{2}$, Samy I. McFarlane ${ }^{1, *}$ \\ ${ }^{1}$ Department of Internal Medicine, State University of New York: Downstate Medical Center, Brooklyn, \\ New York, United States- 11203 \\ ${ }^{2}$ Department of Endocrine Surgery, Kings County Hospital Center, Brooklyn, NY 11203 \\ *Corresponding author: smcfarlane@downstate.edu
}

Received February 24, 2019; Revised April 15, 2019; Accepted June 12, 2019

\begin{abstract}
Colorectal cancer is the third most common cancer in both men and women. Mixed adenoneuroendocrine carcinoma is a rare subtype of colorectal cancer defined as malignant neoplasms in which each component represents at least 30\% of the lesion. It was named as Mixed adenoneuroendocrine carcinoma and described by the World Health Organization in 2010. Due to its morphological heterogeneity and the rarity of the condition, it becomes quite challenging to formulate an appropriate treatment plan. In this report, we present a case of 67-year-old man found to have a rectal mixed adenoneuroendocrine carcinoma. We discussed the current management strategies and provide a review of the literature.
\end{abstract}

Keywords: adeno-neuroendocrine carcinoma

Cite This Article: Aleksander Khudiakov, Mohammed Al-Sadawi. Jia Qin, Michael Haddadin, Stanley Soroka, Angeleque Hartt, Lina Soni, Shalini Arora, and Samy I. McFarlane, "Rare Case of Rectal Mixed Adeno-Neuroendocrine Carcinoma.” American Journal of Medical Case Reports, vol. 7, no. 6 (2019): 117-120. doi: 10.12691/ajmcr-7-6-5.

\section{Introduction}

Colorectal cancer is one of the most common cancers affecting both men and women [1]. It causes almost 694,000 deaths in 2012 [2]. In the world of colorectal carcinoma, mixed adenoneuroendocrine carcinoma (MANEC) is exceptionally rare [3]. It consists of two clinically aggressive neoplasms with considerable morphological heterogeneity which makes it difficult to diagnose and formulate appropriate treatment plan [4]. The criteria for diagnosis are to find each component, adenocarcinoma and neuroendocrine tissue, must comprise at least $30 \%$ of the tumor and both components must be malignant [3]. There is no unifying concesus of how to manage mixed adenoneuroendocrine carcinoma, with some literature suggesting they behave in a similar pattern to more common adenocarcinomas and should be treated as such [5]. Here we present a case of 67-year-old man found to have a rectal mixed adenoneuroedocrine carcinoma.

\section{Report of the Case}

A 67-year-old Hispanic man with significant medical history of eosinophilic esophagitis, 40 pack year tobacco history and alcohol dependence who had a screening colonoscopy performed in 2012 revealing $1.5 \mathrm{~cm}$ sessile polyp in the rectum which was removed, later identified as tubular adenoma and a diminutive rectosigmoid polyp which was difficult to remove due to patient's discomfort. He was referred for a follow up colonoscopy, however, he did not return for it as recommended. He has no family history of gastrointestinal malignancy. In April 2018, he presented to his primary care physician for recurrent urinary tract infection with hematuria. As part of the work up, a CT Urogram was performed revealing an incidental finding of circumferential wall thinking in the distal sigmoid with enlarged adjacent peri-colonic lymph nodes. A later MRI of the pelvis showed diffuse abnormal rectal wall thickening, suspicious for carcinoma along with multiple enlarged perirectal and superior rectal lymph nodes. (Figure 1)

The suspicious mass was evaluated by colonoscopy and a near circumferential mass was found in the rectum concerning for malignancy which was biopsied. Along with mass a $2 \mathrm{~cm}$ rectosigmoid polyp was found and excised (Figure 2). Biopsies of the circumferential mass showed solid sheets and granular architecture with tumor necrosis and desmoplasia; histostaning: synaptophysin positive for more than $50 \%$, chromogranin positive about $1 / 3^{\text {rd }}$, Ki-67 showing $75-90 \%$ nuclear proliferative activity consistent with a Mixed Adenoneuroendocrine Carcinoma (Figure $3 \mathrm{~A}, \mathrm{~B}, \mathrm{C}, \mathrm{D})$. The tumor was staged at IIIA. Carcinoembryonic antigen (CEA) level at the time found to be slightly elevated at $7.1 \mathrm{ng} / \mathrm{ml}(0-4.5 \mathrm{ng} / \mathrm{ml})$ and Chromogranin A levels was $2 \mathrm{nmol} / \mathrm{L}(0-5 \mathrm{nmol} / \mathrm{L})$. A tumor board was held in May 2018 determining that palliative chemoradiation was the appropriate approach 
due to the determination of stage IIIA Mixed Adenoneuroendocrine Carcinoma without symptoms from the mass effect. The type of chemotherapy chosen was made based on the decision to treat this tumor as adenocarcinoma as well as the patient's performance status, which was rapidly declining.

The tumor board recommendation was to treat neuroendocrine component $1^{\text {st }}$ with cisplatin and etoposide followed by chemoradiation using 5- Fluorouracil (5FU). The patient concluded the first two cycles; however, he required an admission to the hospital for rectal pain, hypotension, and leukocytosis. Capecitabine was chosen to replace the 5-Fluorouracil for compliance reasons.

Over the course of several months the patient lost significant weight and his analgesic requirement continued to rise. In October 2018, the patient presented to the emergency room with severe rectal pain and severe constipation with impending total obstipation. Another tumor board was held and concluded that a palliative diverting colostomy is indicated. During his stay another PET scan was performed revealing new multiple subcentimeter pulmonary nodules concerning for metastasis (Figure 4). At the time of the writing of this article the patient was being considered for hospice care.

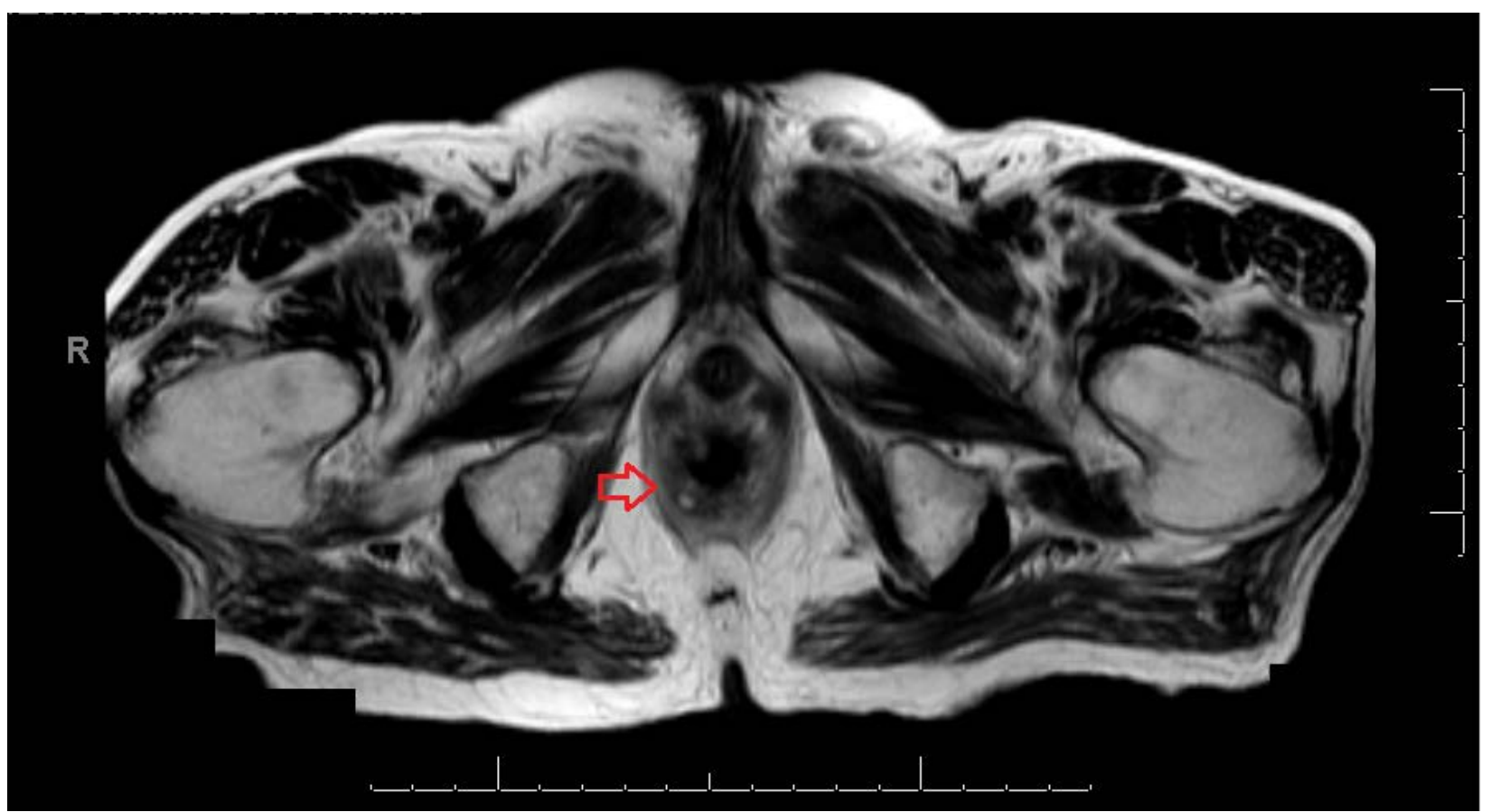

Figure 1. MRI pelvis: diffuse abnormal rectal wall thickening, suspicious for carcinoma along with multiple enlarged perirectal and superior rectal lymph nodes

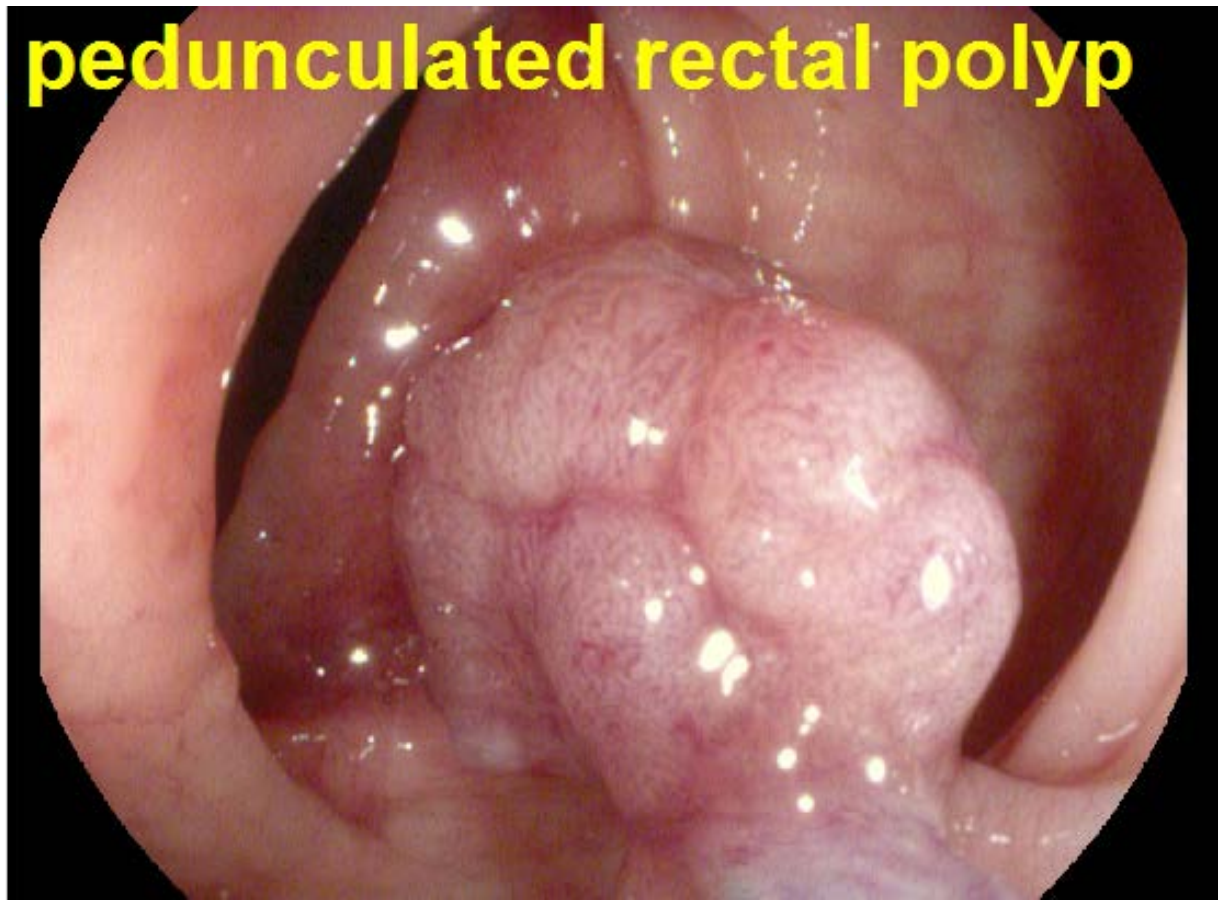

Figure 2. Colonoscopy: pedunculated polyp measuring $2 \mathrm{~cm}$ in size found in the rectum and near circumferential mass was found in the rectum concerning for malignancy. 

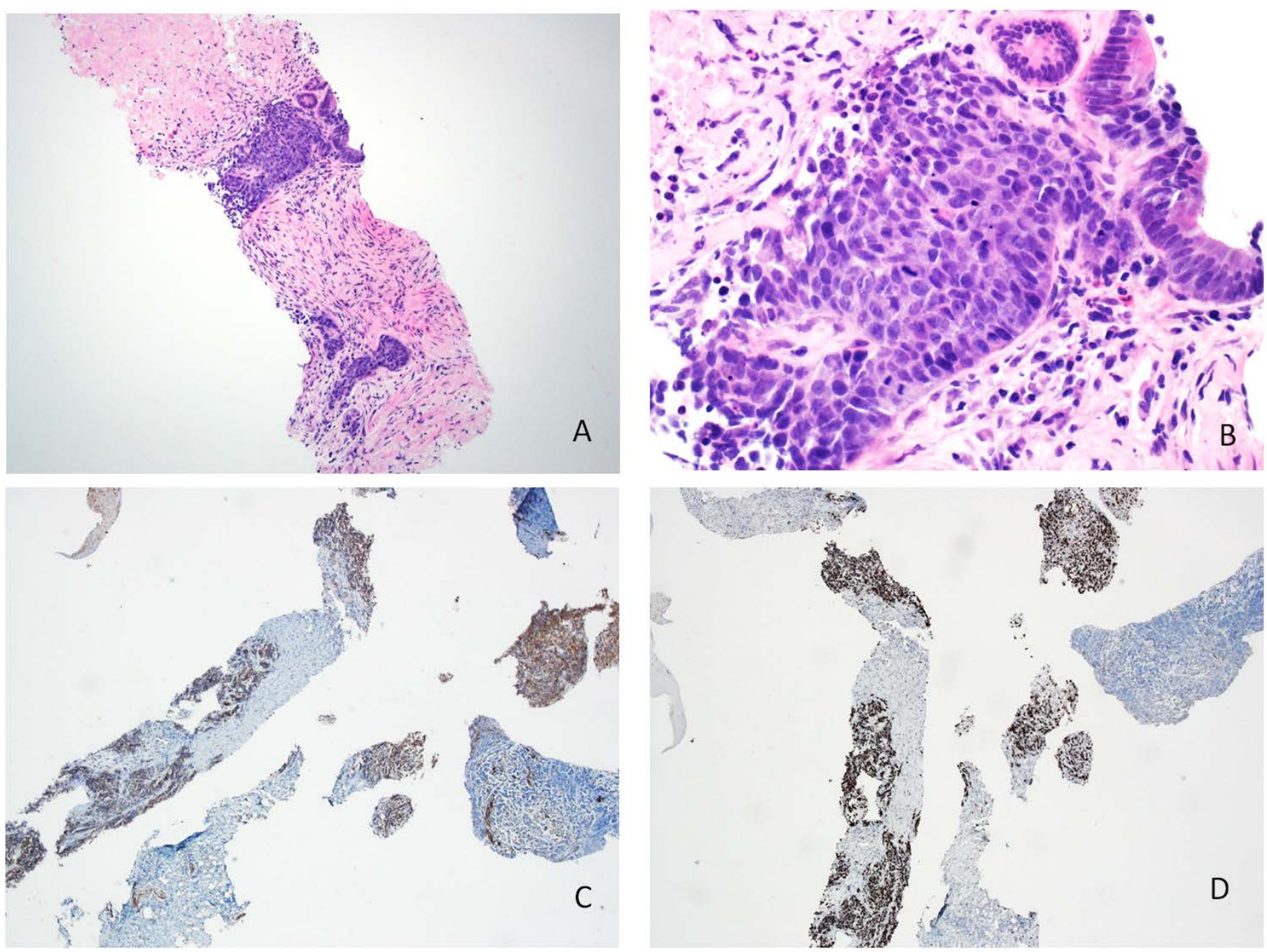

Figure 3. A: H\&P staining at low power (100X), Tumor cells form both solid sheets and granular architecture. The background shows tumor necrosis and dense tumor desmoplasia. B: Adenocarcenoma component H\&P staining at high power (400X), some tumor cells grow in solid sheets. Individual tumor cells are polygonal with high nucleus/cytoplasm ratio, hyperchromatic nuclei with finely granular, stippled chromatin and scant eosinophilic cytoplasm. Nuclear molding is present. Mitotic figures are frequently seen. Adjacent to the tumor nests are well-formed glands. Tumor cells in these glands have uniform, basally oriented and elongated nuclei. Rare intracytoplasmic mucin vacuole is seen. C: Neuroendocrinecomponent. Synaptophysin is positive in more than $65 \%$ of the tumor. $\mathrm{D}$ : Ki-67 is used to evaluate the proliferation index of the tumor. It shows strong positive nuclear proliferation in majority of the tumor cells up to $90 \%$ of the neuroendocrine carcinoma component.

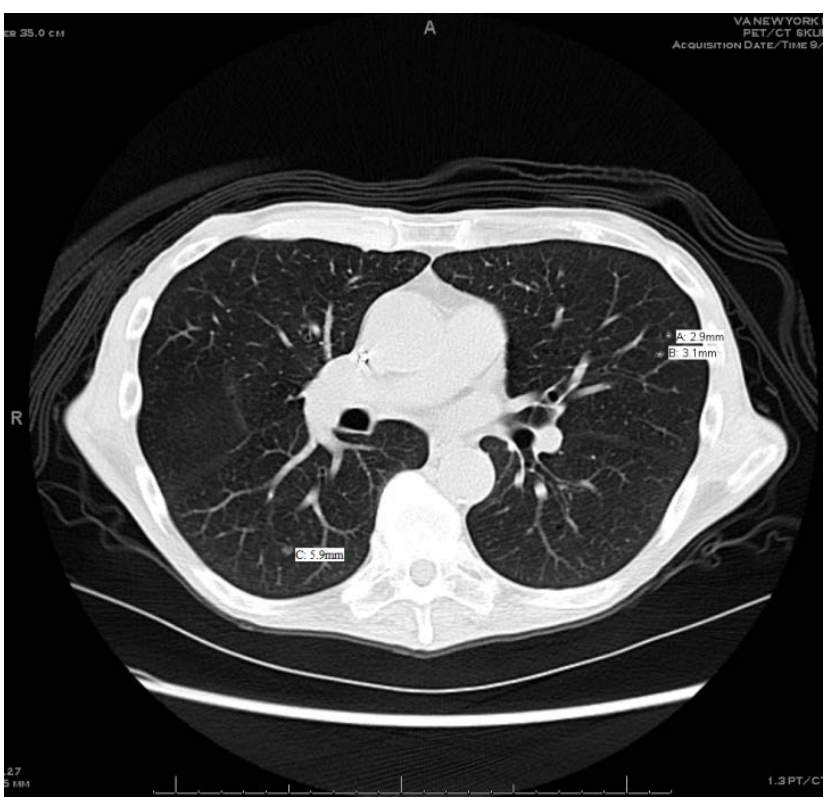

Figure 4. PET scan: new multiple sub centimeter pulmonary nodules concerning for metastasis

\section{Discussion}

Mixed adenoneuroendocrine carcinoma is a rare subtype of colorectal cancer defined as malignant neoplasm in which each component represents at least $30 \%$ of the lesion [3]. It was first described in 1924 [7]; and in 2010, World Health Organization (WHO) described this entity and named it as "mixed adenoneuroendocrine carcinomas” (MANECs) [8]. Macroscopically, MANECs appear, like other colorectal cancers, as polypoid, ulcerating and stenotic circumferential lesions. Histologically, the neuroendocrine part is like small cell or large cell grade 3 neuroendocrine of the lung [3]. The adenocarcinoma component of MANECs can be represented by tubulo-villous or villous adenoma or, in rare occasions, by squamous cell carcinoma [9].

According to WHO 2010 classification [3], two out of three commonly used neuroendocrine markers (synaptophysin, chromogranin A or CD56) must be expressed by immunohistochemical evaluation in the mass to make a diagnosis of MANECs. Synaptophysin is reported as the most sensitive neuroendocrine marker, whereas chromogranin 
A is the most specific [10]. The genetic profile of MANECs was studied; Jesinghaus et al. [3] reported that the genetic and mutation profiles of MANECs is mostly affiliated to adenocarcinoma subtype of colorectal cancer.

Management of MANECs is still an area of debate due to rarity of the condition and lack of strong evidence of the suggested plans. Due to the similarity of genetic and mutation profiles of MANECs to adenocarcinoma, it was suggested to treat MANECs as adenocarcinoma [3,11]. This treatment plan would consist of the standard FLOFOX(5-FU-lucovorin-oxycysplatin) therapy along with surgical reception if possible which was shown successful in case reports.

In case of poorly differentiated neuroendocrine component of MANECs, treating as neuroendocrine malignancy should be considered [12]. In these cases, an approach targeting neuroendocrine component might be favored, such as using a platinum agent (carboplatin or cisplatin) along with etoposide. 5-FU can be added for the adenocarcinoma component. As we shown with our case this approach has significant side effects and may be difficult for patients to comply with especially in rapidly progressive tumors with rapid functional decline.

The prognosis of MANECs varies according to site, stage and grade. In colorectal MANECs, no survival rate difference was found compared to neuroendocrine [11]. However, gastric and esophageal MANECs were found to have a better survival rates compared to pure neuroendocrine tumors [13]. More investigation needs to be conducted into a standardize approach to patient with MANEC, however it is difficult to accumulate significant studies and case reports due to the high variability among tumors.

\section{Acknowledgements}

This work is supported, in part, by the efforts of Dr. Moro O. Salifu M.D., M.P.H., M.B.A., M.A.C.P., Professor and Chairman of Medicine through NIH Grant number S21MD012474.

\section{References}

[1] Ferlay J, Soerjomataram I, Dikshit R, Eser S, Mathers C, Rebelo M, Parkin DM, Forman D, Bray F. Cancer incidence and mortality worldwide: sources, methods and major patterns in GLOBOCAN 2012. Int J Cancer. 2015 Mar 1; 136(5): E359-86.

[2] Brenner H, Kloor M, Pox CP. Colorectal cancer. Lancet. 2014 Apr 26; 383(9927):1490-1502.

[3] Bosman FT, Carneiro F, Hruban RH, THeise ND, eds. WHO Classification of Tumours of the Digestive System, 4th Edition. IARC Press, Lyon, 2010 pp. 13-4.

[4] Düzköylü Y, Aras O, Bostancı EB, Temuçin TK, Ulaş M. Mixed Adeno-Neuroendocrine Carcinoma; Case Series of Ten Patients with Review of the Literature. Balkan Med J. 2018 May; 35(3): 263-267.

[5] Jesinghaus M, Konukiewitz B, Keller G, Kloor M, Steiger K, Reiche $\mathrm{M}$, et al. Colorectal mixed adenoneuroendocrine carcinomas and neuroendocrine carcinomas are genetically closely related to colorectal adenocarcinomas. Mod Pathol. 2017 Apr; 30(4):610-619.

[6] Jess T, Rungoe C, Peyrin-Biroulet L. Risk of colorectal cancer in patients with ulcerative colitis: a meta-analysis of populationbased cohort studies. Clin Gastroenterol Hepatol. 2012 Jun; 10(6): 639-45.

[7] Cordier R. Les cellules argentaffines dans les tumeurs intestinales. Arch. Int. Med. Exp. 1924; 1: 5.

[8] Rindi G., Arnold R., Bosman F.T., Capella C., Kilmstra D.S Kloppel G., Komminoth P., Solcia E. Nomenclature and classification of neuroendocrine neoplasms of the digestive system. In: Bosman F.T., Carneiro F., Hruban R.H., Theise N.D., editors. WHO Classification of Tumours of the Digestive System. 4th. IARC Press; Lyon, France: 2010. pp. 13-14.

[9] La Rosa S, Marando A, Furlan D, Sahnane N, Capella C. Colorectal poorly differentiated neuroendocrine carcinomas and mixed adenoneuroendocrine carcinomas: insights into the diagnostic immunophenotype, assessment of methylation profile, and search for prognostic markers. Am J Surg Pathol. 2012 Apr; 36(4): 601-11.

[10] Klimstra DS, Modlin IR, Adsay NV, et al. Pathology reporting of neuroendocrine tumors: application of the Delphic consensus process to the development of a minimum pathology data set. Am J Surg Pathol. 2010; 34(3): 300-313.

[11] La Rosa S, Marando A, Sessa F, Capella C. Mixed Adenoneuroendocrine Carcinomas (MANECs) of the Gastrointestinal Tract: An Update. Cancers (Basel). 2012;4 (1): 11-30. Published 2012 Jan 16.

[12] Hervieu V, Scoazec JY. [Mixed endocrine tumors]. Ann Pathol. 2005 Dec; 25(6): 511-28.

[13] Maru DM, Khurana H, Rashid A, Correa AM, Anandasabapathy S Krishnan S, Komaki R, Ajani JA, Swisher SG, Hofstetter WL. Retrospective study of clinicopathologic features and prognosis of high-grade neuroendocrine carcinoma of the esophagus. Am J Surg Pathol. 2008 Sep; 32(9): 1404-11. 